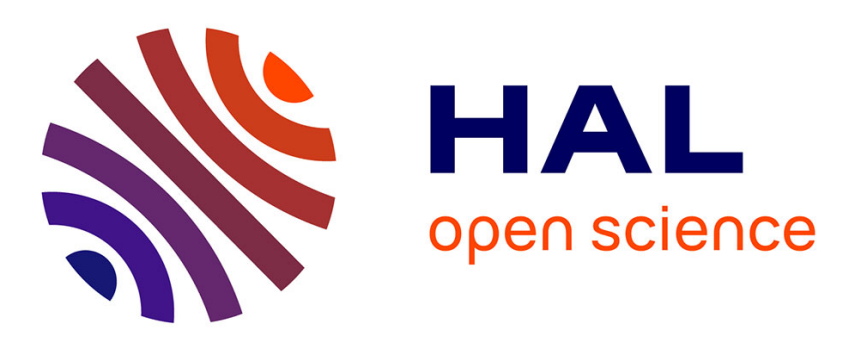

\title{
Mechanisms of remanent optical alignment of dyes in a gel matrix
}

Florian Bentivegna, Michael Canva, Alain Brun, Frédéric Chaput, Jean-Pierre Boilot

\section{- To cite this version:}

Florian Bentivegna, Michael Canva, Alain Brun, Frédéric Chaput, Jean-Pierre Boilot. Mechanisms of remanent optical alignment of dyes in a gel matrix. Journal of Applied Physics, 1996, 80 (8), pp.4655-4659. 10.1063/1.363425 . hal-00668876

\section{HAL Id: hal-00668876 \\ https://hal-iogs.archives-ouvertes.fr/hal-00668876}

Submitted on 10 Feb 2012

HAL is a multi-disciplinary open access archive for the deposit and dissemination of scientific research documents, whether they are published or not. The documents may come from teaching and research institutions in France or abroad, or from public or private research centers.
L'archive ouverte pluridisciplinaire HAL, est destinée au dépôt et à la diffusion de documents scientifiques de niveau recherche, publiés ou non, émanant des établissements d'enseignement et de recherche français ou étrangers, des laboratoires publics ou privés. 


\section{AIP $\mid$ Applied Physics}

\section{Mechanisms of a remanent optical alignment of dyes in a gel matrix}

Florian Bentivegna, Michael Canva, Alain Brun, Frédéric Chaput, and JeanPierre Boilot

Citation: J. Appl. Phys. 80, 4655 (1996); doi: 10.1063/1.363425

View online: http://dx.doi.org/10.1063/1.363425

View Table of Contents: http://jap.aip.org/resource/1/JAPIAU/v80/i8

Published by the American Institute of Physics.

\section{Related Articles}

Spin-dependent synchrotron $\mathrm{x}$-ray excitations studied by scanning tunneling microscopy J. Appl. Phys. 111, 07E304 (2012)

$1 s 2 p$ resonant inelastic $x$-ray scattering-magnetic circular dichroism: A sensitive probe of $3 \mathrm{~d}$ magnetic moments using hard $\mathrm{x}$-ray photons

J. Appl. Phys. 111, 07E301 (2012)

High magnetic field annealing effect on visible photoluminescence enhancement of $\mathrm{TiO} 2$ nanotube arrays

Appl. Phys. Lett. 100, 043106 (2012)

Enhancement of the transverse non-reciprocal magneto-optical effect

J. Appl. Phys. 111, 023103 (2012)

Induced magnetic anisotropy and spin polarization in pulsed laser-deposited Co2MnSb thin films

J. Appl. Phys. 111, 023903 (2012)

\section{Additional information on J. Appl. Phys.}

Journal Homepage: http://jap.aip.org/

Journal Information: http://jap.aip.org/about/about_the_journal

Top downloads: http://jap.aip.org/features/most_downloaded

Information for Authors: http://jap.aip.org/authors

\section{ADVERTISEMENT}

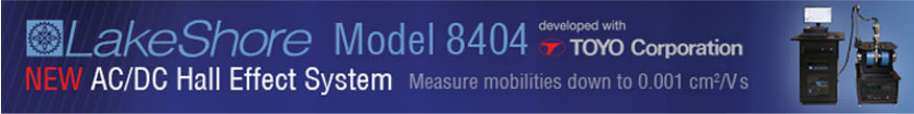




\title{
Mechanisms of a remanent optical alignment of dyes in a gel matrix
}

\author{
Florian Bentivegna, Michael Canva, and Alain Brun \\ Groupe d'Optique Non Linéaire, URA CNRS 14, Institut d'Optique Théorique et Appliquée, \\ Centre Universitaire d'Orsay, 91403 Orsay Cedex, France \\ Frédéric Chaput and Jean-Pierre Boilot \\ Laboratoire de Physique de la Matière Condensée, URA CNRS 1254D, Ecole Polytechnique, \\ 91128 Palaiseau Cedex, France
}

(Received 3 April 1996; accepted for publication 8 July 1996)

\begin{abstract}
We present the mechanisms of a remanent alignment of polarizable molecules dispersed in a solid silica gel matrix, when submitted to powerful, femtosecond, linearly polarized, optical pulses emitted from a dye laser. This alignment can be related to third-order nonlinearities of the dopants through an orientational optical Kerr effect. Its remanence results from the strong interactions the dopants share with their host matrix, particularly through hydrogen bonds. We expose here the detailed mechanism of this phenomenon, considering that such a rotation of large chromophores may not be compatible at first sight with subpicosecond pulse durations. Through several experiments, we show that this apparent contradiction is solved once analyzed, by the structure of the excitation pulses, which cannot be simply described as "femtosecond pulses", but consist of two temporal components-a femtosecond one and a nanosecond one-playing different but essential roles in the process. () 1996 American Institute of Physics. [S0021-8979(96)00520-8]
\end{abstract}

\section{INTRODUCTION}

Solid matrices prepared by a sol-gel process and doped by various optically active species have proved to be highly interesting materials for nonlinear optics. ${ }^{1-3}$ Within the past few years, we have shown that such media could present an original behavior when submitted to ultrashort optical pulses. ${ }^{4,5}$ If trapped in a sufficiently dense gel matrix, ordinary optically nonlinear chromophores can lead to notable and persistent birefringences under the influence of linearly polarized, subpicosecond, light pulses-the birefringence principal axis corresponding to the very polarization direction of the pulses.

As previously reported, such an alignment of polarizable molecules under the influence of ultrashort light pulses can be attributed to their third-order nonlinear optical properties. Moreover, its temporal persistence arises from the strong interactions the molecules share with their host matrix. Still, the detailed mechanism of this optical alignment remained only partially understood, since such a mechanical rotation of large molecules does not appear to be compatible with subpicosecond pulse durations. We show here that this phenomenon can be explained by the actual temporal structure of the exciting pulses, which appears more complex than expected due to the generation technique of these light pulses. We establish that they bear two temporal components, each one playing a different but essential role in the optical alignment process.

\section{OPTICAL MEMORY EFFECT IN DOPED GEL MATRICES}

Polarizable chromophores (and especially organic molecules such as rhodamines or azo-dyes) trapped in the pores of a silica gel matrix can be locally aligned when they are subjected to subpicosecond, linearly polarized, optical pulses. ${ }^{4}$ This alignment of doping chromophores shows a remarkable remanence even after the exciting pulse source has been switched off. We could thus describe the phenomenon as an all-optical memory effect, since the photoinduced birefringence is also "read" through a cw $\mathrm{He}-\mathrm{Ne}$ laser beam.

The excitation pulses are issued from a classical colliding pulse, passively mode-locked dye resonator, and then amplified through several dye stages pumped by a frequency-doubled Nd:YAG laser. Several experiments led us to attribute this birefringence to an optical Kerr effect taking place in each molecule and responsible for an overall alignment of the dopants towards the direction of the optical exciting electric field.

Moreover, the persistence of this birefringence seems to be due to the numerous hydrogen bonds that can be established between the doping molecules and their host matrix. Hydrogen bonds are sufficiently strong to overcome the thermal agitation at normal temperature and maintain the molecules in a given position in the absence of any external excitation. Yet they are also weak enough to be broken by powerful light pulses. Between each pulse, new hydrogen bonds can be formed and can freeze in the directions adopted by the dopants. Pulse after pulse, these molecular directions tend to align towards the polarization direction of light. Once the excitation pulses have been switched off, most molecules keep their induced direction. Thus the orientational anisotropy can remain at a high level for very long times. For example, images we inscribed in such doped gel matrices remain legible without ambiguity more than three years after their inscription.

Both the molecular motion and the conservation of photoinduced molecular directions can be described in terms of rotational diffusion. ${ }^{6,7}$ Yet mechanical rotation of large dopants such as rhodamine molecules cannot occur during typical subpicosecond pulses. Still all our experimental results showed a clear dependence of the phenomenon on the 
optical power and not on the optical energy. For example, no birefringence could be observed with nanosecond pulses of even 100 times higher energy. This means that the subnanosecond duration of the pulses is an essential parameter of the phenomenon. On the other hand, the induced optical transmission coefficient appeared to be strictly linear with respect to the pulse energy, which excludes any multiphoton effect. The solution to this dilemma may be hidden in the real, and maybe not so simple, temporal structure of the optical pulses we used.

\section{TEMPORAL STRUCTURE OF THE EXCITATION PULSES}

As previously mentioned, these optical pulses are emitted from a colliding pulse, passively mode-locked (CPM) ring resonator pumped by a cw Ar laser. Obtained at a mean wavelength of about $620 \mathrm{~nm}$ (the amplification medium is a rhodamine $6 \mathrm{G}$ jet stream), they are emitted at a high repetition rate of $80 \mathrm{MHz}$ for a typical duration of 150-200 fs. Yet they still are not energetic enough $(0.1 \mathrm{~nJ})$ to produce any nonlinear effect on classical chromophores, and must be amplified. This amplification takes place through four circulating dye solution stages pumped by a frequency-doubled $\mathrm{Nd}$ :YAG laser.

Thus immediately after their exit from the oscillating ring cavity, the pulses essentially show a subpicosecond duration. But this is no longer the case after they travel through the four amplification stages. Each one of these stages is made of a sulforhodamine 640 solution pumped at $532 \mathrm{~nm}$, near its absorption maximum $\lambda_{\max }$. Part of the pump energy is lost by radiative deexcitation of the sulforhodamine 640 molecules in the first amplification stage, which induces a notable fluorescence signal. Such a fluorescence signal is emitted in all directions, and a fraction of it travels in the same direction as the amplified subpicosecond pulse. This fluorescence radiation is then amplified in the next three dye stages. The duration of this signal is approximately equal to the duration $\Delta t$ of the Nd:YAG pump pulses (i.e., about 8 ns), since typical fluorescence time constants of such dyes are of the same range as $\Delta t$.

Thus the temporal structure of our exciting pulses becomes more complex after being amplified. We can describe it as an ultrashort femtosecond component (with a typical energy of $10 \mu \mathrm{J})$, on which a much longer nanosecond one is superimposed (Fig. 1). The energy of the longest component is typically $5 \%-10 \%$ that of the (amplified) femtosecond one, but can be made, if required, comparable to it or even higher (up to $100 \mu \mathrm{J}$ ). Still the optical power of the longest component is, of course, much weaker than that of the shortest one, at least by six orders of magnitude. This comparison stands for the corresponding optical electric fields, with a ratio of about three orders of magnitude.

On this basis, we formulated the following hypothesis, attributing complementary roles to each of the two temporal components. First, the highly powerful, femtosecond component would induce a strong dipolar moment in each molecule. Thus a large torque would break the hydrogen bond(s) between the molecule and its matrix. Then the nanosecond component would induce weaker dipolar moment and

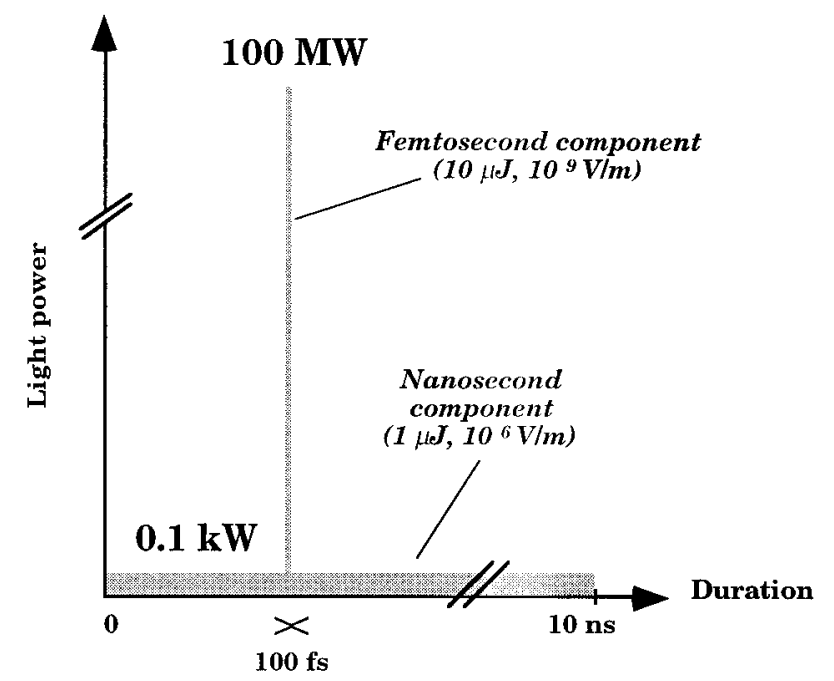

FIG. 1. Schematic representation of the temporal structure of the excitation pulses.

torque. This torque would be strong enough, nevertheless, to attract the molecule towards the direction of the optical electric field. Several experiments were carried out in order to confirm this hypothesis.

\section{EXPERIMENTAL STUDY}

For that purpose, the ideal experimental situation would be of course to use "pure" femtosecond pulses (i.e., with no fluorescence nanosecond component) emitted at the same wavelength as our excitation pulses.

As reported above, we could easily obtain pure nanosecond pulses from our laser source by interrupting the femtosecond pulses issued from the CPM. Then the sole nanosecond fluorescence pulses produced in the amplification stages are used. We showed that such pulses did not induce any measurable birefringence.

On the contrary, it is not possible to produce absolutely pure femtosecond pulses with the same energy as in the twocomponent exciting pulses, and totally separated from the nanosecond component. Typical response times of optical switches are much too long for that purpose. In the same way, optical insulators cannot be used here, since both temporal components are in the same state of polarization.

We got around this difficulty by superimposing auxiliary nanosecond pulses on our two-component main excitation pulses. The idea is that these nanosecond auxiliary pulses could reveal, by modifying it, the role of the nanosecond component of the main excitation pulses. These auxiliary pulses were obtained from a circulating sulforhodamine 640 reservoir pumped by a frequency-doubled Nd:YAG laser (the same one that also pumps the amplification stages described above). They present the same wavelength $(620 \mathrm{~nm})$ and duration ( $8 \mathrm{~ns}$ ) as the nanosecond component of the excitation pulses. Auxiliary and excitation pulses are emitted in perfect synchrony but can be independently polarized. Auxiliary pulses can also be delayed with respect to the excitation pulses by using a delay line. 


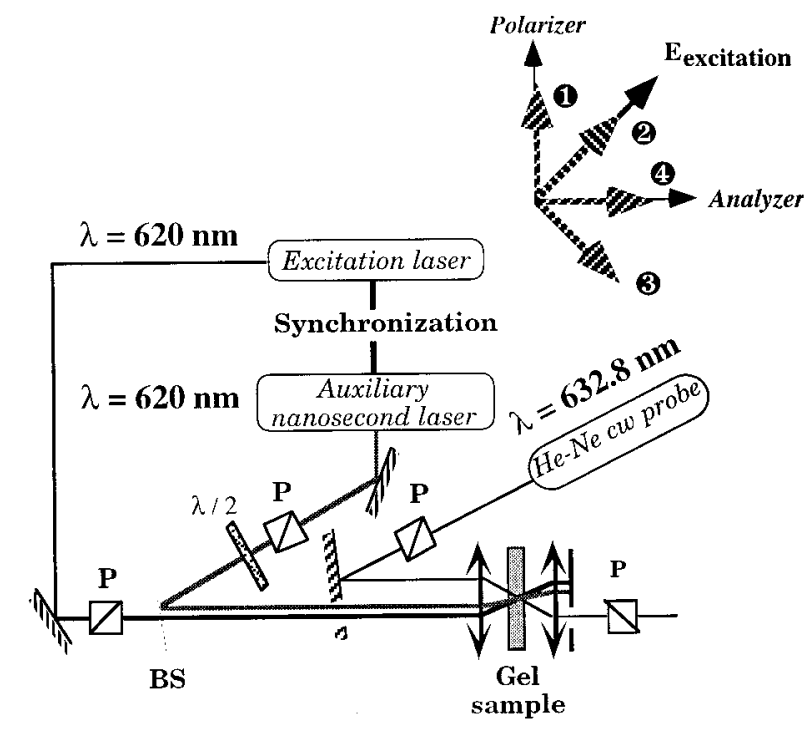

FIG. 2. Experimental setup for the study of the respective roles of the nanosecond and femtosecond components of the excitation pulses. (P) polarizers and (BS) - beamsplitter. The inset shows the different configurations for the auxiliary nanosecond pulse polarization.

Doped gel samples were placed between crossed polarizers, so that the light issued from a cw He-Ne probe laser is not transmitted as long as the sample remains isotropic. Two-component main excitation pulses were polarized at $45^{\circ}$ from the crossed polarizers to ensure a maximum transmission signal when the sample becomes anisotropic. Four polarization directions of the auxiliary pulses were studied (Fig. 2): (1) parallel to the polarizer placed before the sample; (2) parallel to the main excitation pulses; (3) orthogonal to the main excitation pulses; and (4) parallel to the analyzer placed after the sample.

In addition, we noted " 0 ," the situation where the host molecules are submitted to the sole excitation pulses.

All three laser beams (main excitation pulses, auxiliary pulses, and probe beam) were focused on the sample with a spot diameter of about $100 \mu \mathrm{m}$. Note that the probe spot must be a little bit smaller than the two others, in order to ensure homogeneous detection of the induced birefringences. Experiments were carried out on a 1-mm-thick, mixed silica/ zirconia gel, with a molar ratio equal to $7: 1$, doped with rhodamine 640 molecules at a concentration of $4.10^{-4} \mathrm{~mol}$ $l^{-1}$ in the sol. ${ }^{5}$ The pore size in such matrices depends directly on the size of the dopants, since the gel network grows around the dopants during the synthesis. We estimate here the average pore size to be nanometric.

Let us note finally that, considering the size of the optical spots on the sample, it is extremely difficult to ensure that the superposition of the three spots is exactly the same in all cases studied. For that reason, and also because of slight thickness nonuniformities of the sample and of possible excitation energy fluctuations, we preferred not to quantitatively compare the experimental results. Thus in the following, comparisons between the different experimental configurations are essentially qualitative.

We first compared the birefringence measured in each of the four configurations to the birefringence obtained with the sole main excitation pulses (config. 0). Figure 3 shows that superimposing the auxiliary nanosecond pulses reduces the induced birefringence in three of the four cases (configs. 1, 3, and 4). On the contrary, it enhances the birefringence in configuration 2. Moreover, in all cases, the sign of the photoinduced birefringence $\Delta n$ remains the same as in configuration 0 , as confirmed using a Soleil-Bravais compensator. Let us also note the importance of the temporal offset between auxiliary and excitation pulses. The delay line mentioned above must be adjusted so that the auxiliary pulses reach the sample in the same time as or slightly later than the excitation ones. No modification in the induced birefringence was observed when the auxiliary nanosecond pulses were set ahead of the excitation pulses.

The energy of the auxiliary pulses also plays an important role in the process. Figure 3 shows that the modification (reduction or enhancement) of the measured birefringence due to these pulses increases with their energy. This energy (from $1 \mu \mathrm{J}$ up to about $30 \mu \mathrm{J}$ ) must be compared to the typical energies of the two components of the main excitation pulses: $10 \mu \mathrm{J}$ for the femtosecond component and $1 \mu \mathrm{J}$ for the nanosecond one.

The corrective orientation effect of the auxiliary pulses also clearly appears when these are switched on some time after the excitation pulses. Figure 4 shows this effect in the case of configuration 1. Three molecular alignment kinetics are represented in Figs. 4(a)-4(c): with the sole main excitation pulses, with both excitation and auxiliary pulses switched on at the same time, and with "delayed" (i.e., switched on about 4 min after the main excitation pulses) auxiliary pulses, respectively. In the last case, we can observe without ambiguity a short transient period during which the birefringence decreases, followed by an increase that asymptotically tends to the kinetics of the case of Fig. 4(b). Let us also note that the same observation was made for configurations 3 and 4 , and that $\Delta n$, on the contrary, increased in configuration 2 when the auxiliary pulses were switched on.

\section{DISCUSSION}

We can now examine these experimental observations from the perspective of the hypothesis we made. The molecular alignment kinetics obtained in each of the four configurations (Fig. 3) coincide fairly well with the respective roles we attributed to the two temporal components of the excitation pulse.

Thus in configurations 1 and 4, the auxiliary nanosecond pulses tend to align the molecular dipoles along one of the neutral axes of the two polarizers placed on both sides of the sample. In doing so, they partly counterbalance the action of the excitation pulses and especially their nanosecond component that attracts the molecules $45^{\circ}$ from the neutral axes. These contradictory tendencies logically result in a decrease of the induced birefringence, compared to configuration 0 .

Similarly, in configuration 3 , the auxiliary pulses oppose molecular alignment due to the excitation pulses. Here the two competitive preferential directions are perpendicular to one another, and the resulting birefringence gets weaker. 


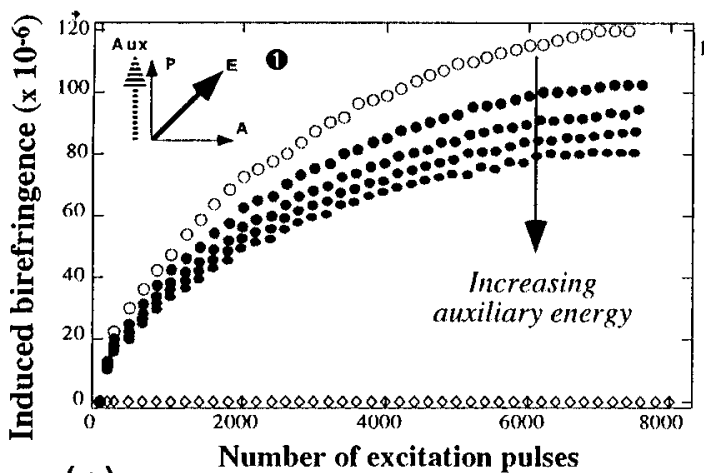

(a)

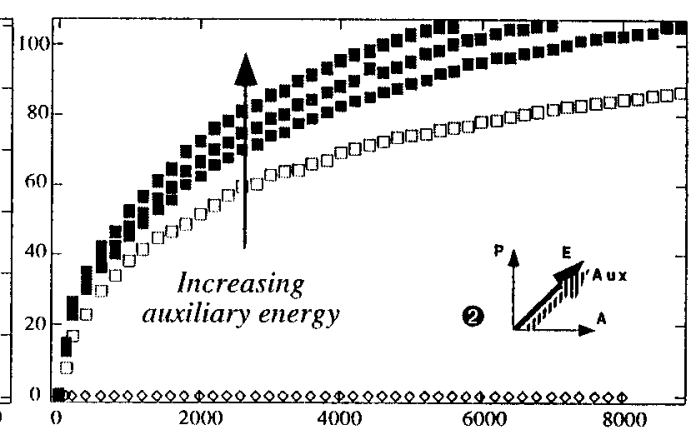

(b)
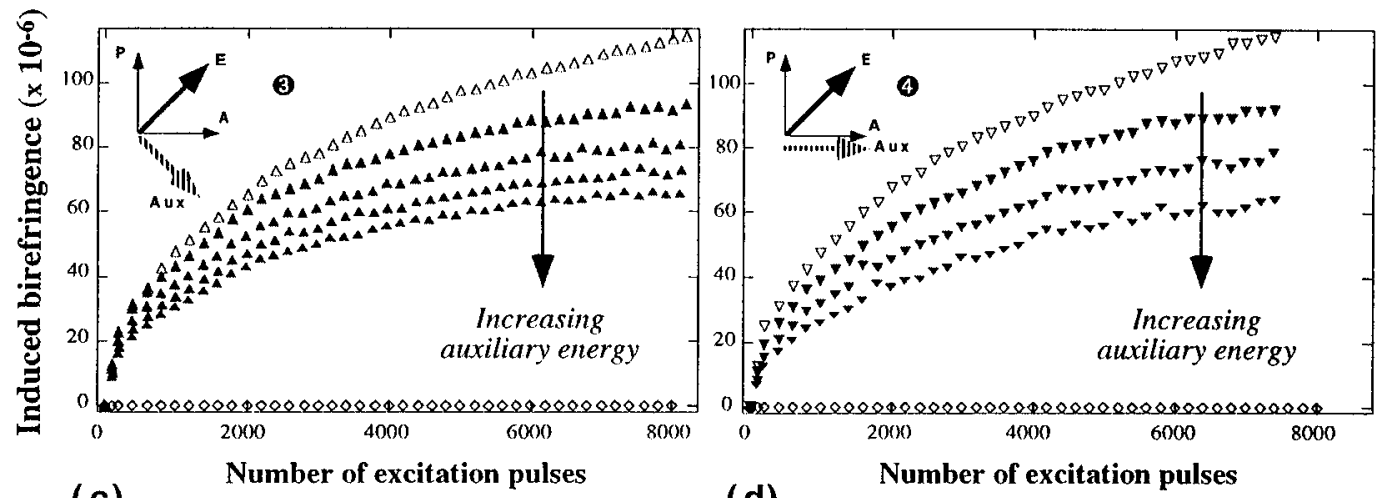

(c)

(d)

FIG. 3. Corrective effects on the molecular alignment kinetics due to the superposition of auxiliary pulses (for different energies), as a function of the number of excitation pulses. Open symbols represent the kinetics without auxiliary pulses (i.e., with the sole excitation pulses), and closed symbols the kinetics with auxiliary pulses. Losanges figure the response of the sample submitted to the sole auxiliary pulses. (P) and (A) — polarizers placed before and after the sample. (E) and (Aux) — the respective polarizations of the excitation and auxiliary pulses, respectively.

On the contrary, in configuration 2 , both auxiliary and excitation pulses are in the same state of polarization and they work together. In this case, the induced birefringence is larger than that of configuration 0 , as confirmed by our measurements.

The importance of a temporal quasicoincidence of excitation and auxiliary pulses in the sample can also be explained according to our model. The essential part of the

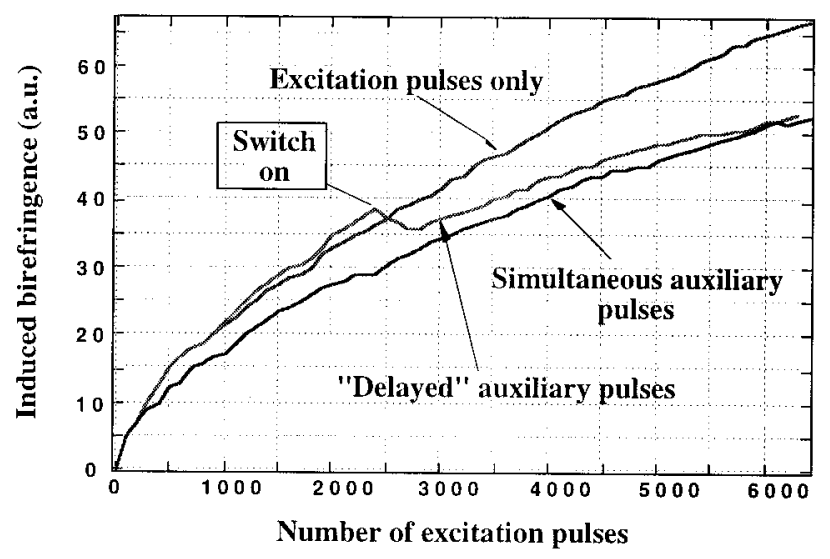

FIG. 4. Comparison of the molecular alignment kinetics obtained (in config. 1) with the sole main excitation pulses (a), with both excitation and auxiliary pulses switched on at the same time (b), and with "delayed" (switched on 4 min after the excitation pulse train) auxiliary pulses (c). A transient period is observed in (c). energy carried by the nanosecond auxiliary pulse must reach the sample exactly when (or slightly after) the femtosecond component of the excitation pulse liberates the molecules, and before new hydrogen bonds can be formed. In the opposite case, we observed that the auxiliary pulse alone does not induce any corrective effect to the molecular anisotropy. This corroborates the role played by the nanosecond component of the excitation pulse in the molecular alignment process. In the same way, this role is also confirmed by the growing corrective effect induced by the auxiliary pulses when their energy increases. The molecular alignment is made easier and quicker when the mechanical torque exerted by the nanosecond electric field gets larger.

Similarly, the observation of a transient period with delayed auxiliary pulses (Fig. 4) meets the terms of our hypothesis. In the configuration showed here (config. 1), the auxiliary pulses tend to oppose the action of the shear excitation pulses. This explains why the birefringence temporarily decreases. This experiment also clearly establishes that the auxiliary pulses (like in the preceding experiments) exert a corrective but not directive effect. The excitation pulses always keep their dominating role in the alignment process, and the transient period is short; the birefringence rapidly increases again, reaching the level observed in the case of Fig. 4(b) (with both excitation and auxiliary pulses).

Still one may think that the auxiliary nanosecond pulses could somehow oppose the molecular anisotropy or even to- 
tally reverse the evolution of the birefringence. To that end, the auxiliary pulses should act after the molecules have been liberated by the femtosecond component of the main excitation pulses, and while they get aligned by the nanosecond component. This alignment takes place during a short period that can be estimated as a few hundred picoseconds after the end of the femtosecond component (the approximate time necessary to break or form a hydrogen bond) but it remains difficult to determine precisely.

\section{CONCLUSION}

In conclusion, all the experimental observations we gathered and presented in this article appear to find a logical and natural explanation in our hypothesis. To sum up, we established the role of the nanosecond-range component of the femtosecond light pulses used for aligning molecular dipoles dispersed in the silica gel matrix we studied. This component alone is still not sufficient to get to this result, since the doping molecules first need to be liberated from the silica network. This requires a powerful, femtosecond-range pulse component. It may seem then that the nonlinear phenomenon we describe here in terms of optical memory could not have been observed without the type of laser source we use in our laboratory. Yet this kind of laser remains one of the most common femtosecond sources that can be found in research laboratories nowadays, especially at visible wavelengths. All the experiments we described above show that the nanosecond component present in the pulses emitted from these lasers cannot systematically be neglected in interpreting the nonlinear optical effects they induce. This holds not only for the memory optical Kerr effect but also for various other nonlinearities such as saturation absorption, reverse saturable absorption, etc. It is possible that sufficiently energetic picosecond pulses might be able to induce a memory optical Kerr effect in our samples. Further experiments need to be carried out in order to confirm this prediction.

${ }^{1}$ Sol-Gel Optics I, edited by J. D. Mackenzie (The International Society for Optical Engineering, San Diego, 1990), Vol. 1328, and references therein.

${ }^{2}$ Sol-Gel Optics II, edited by J. D. Mackenzie (The International Society for Optical Engineering, San Diego, 1992), Vol. 1758, and references therein.

${ }^{3}$ Sol-Gel Optics III, edited by J. D. Mackenzie (The International Society for Optical Engineering, San Diego, 1994), Vol. 2288 and references therein.

${ }^{4}$ M. Canva, G. Le Saux, P. Georges, A. Brun, F. Chaput, and J. P. Boilot, Opt. Lett. 17, 218 (1992).

${ }^{5}$ F. Bentivegna, M. Canva, A. Brun, F. Chaput, and J. P. Boilot, in Sol-Gel Optics III, edited by J. D. Mackenzie (The International Society for Optical Engineering, San Diego, 1994), Vol. 2288, p. 609.

${ }^{6} \mathrm{H}$. Benoît, Ann. Phys. (France) 6, 561 (1951).

${ }^{7}$ P. Pernot, R. Pansu, B. Levy, and J. Faure, Chem. Phys. 177, 793 (1993). 